\title{
Concomitant Partial Achilles Tendon Rupture and Lateral Luxation of the Superficial Digital Flexor Muscle Tendon in a Dog
}

\author{
Hélène Dosseray ${ }^{1} \quad$ Claire Deroy-Bordenave ${ }^{2}$ \\ ${ }^{1}$ Department of Orthopaedics and Neurology, Fitzpatrick Referrals, \\ Halfway Lane, Eashing, Godalming, Surrey, United Kingdom \\ 2 Vet and Surg, Rue Sauternes, Le Haillan, France \\ VCOT Open 2021;4:e37-e40.
}

\begin{abstract}
Address for correspondence Hélène Dosseray, DVM, GPCert(SAS), Department of Orthopaedics and Neurology, Fitzpatrick Referrals, Halfway Lane, Eashing, Godalming, Surrey, GU7 2QQ, United Kingdom

(e-mail: h.dosseray@gmail.com).
\end{abstract}

\begin{abstract}
Keywords

- dogs

- Achilles tendon rupture

- superficial digital flexor muscle tendon

- luxation

The aim of this study was to report a posttraumatic partial Achilles tendon (AT) rupture associated with lateral luxation of the superficial digital flexor tendon (SDFT) in a Whippet. This article is a brief communication. A Whippet was presented with posttraumatic plantigrade stance and non-load-bearing lameness of the right pelvic limb. The objective findings consisted in partial AT rupture and SDFT lateral luxation. Surgical treatment ensued: tenorrhaphy of the torn tendons and calcaneo-tibial screw insertion for tarsal immobilization, followed by suturing of the SDFT retinaculum. A casting bandage was employed for additional immobilization. Nonetheless, a bandage complication prompted the premature removal of the fixation screw and casting wrap. Complete functional recovery was achieved by the 20th postoperative week. The simultaneous occurrence of SDFT luxation and partial AT tear has not been reported in the literature before. The long-term postoperative functional outcome was highly satisfactory.
\end{abstract}

\section{Introduction}

Partial rupture of the Achilles tendon (AT) and lateral luxation of the superficial digital flexor muscle tendon (SDFT) are two distinct pathologies. Partial AT rupture is more frequent than SDFT luxation. ${ }^{1,2}$ The AT originates from various musculotendinous units: (1) the SDFT, (2) the gastrocnemius muscle tendon, and (3) the common tendon of the gracilis, biceps femoris, and semitendinosus muscles. ${ }^{1,3}$ The aetiology of AT injuries in the dog is most often traumatic. ${ }^{1}$ Achilles tendon injuries may also be secondary to systemic disease or drug-induced. ${ }^{1}$ The severity of injury may vary according to the origin and nature of trauma. ${ }^{1}$ The SDFT is the tendon component that often remains intact in partial AT ruptures. ${ }^{3}$ Luxation occurs because of tear in the tendon's medial or lateral retinaculum on the calcaneal tuberosity, the lateral

received

November 9, 2020

accepted after revision

March 29, 2021 luxation variant being the more common of the two. ${ }^{2}$ Trauma or hereditary structural abnormalities of the SDFT groove on the calcaneal tuberosity have been reported as predisposing factors. $^{2}$ The concomitant occurrence of the two injuries (SDFT luxation and partial AT tear) has not hitherto been reported.

\section{Case Description}

An ovariectomized 11-year-old $15 \mathrm{~kg}$ (2/5 body score condition) female Whippet was presented with plantigrade stance and non-load-bearing lameness of the right hindlimb, which had been inflicted a day earlier during a traumatic encounter with another dog. The owners did not report any previous medical or orthopaedic problems up to this injury. General

(C) 2021. The Author(s).

This is an open access article published by Thieme under the terms of the Creative Commons Attribution License, permitting unrestricted use, distribution, and reproduction so long as the original work is properly cited. (https://creativecommons.org/licenses/by/4.0/)

Georg Thieme Verlag KG, Rüdigerstraße 14, 70469 Stuttgart, Germany 


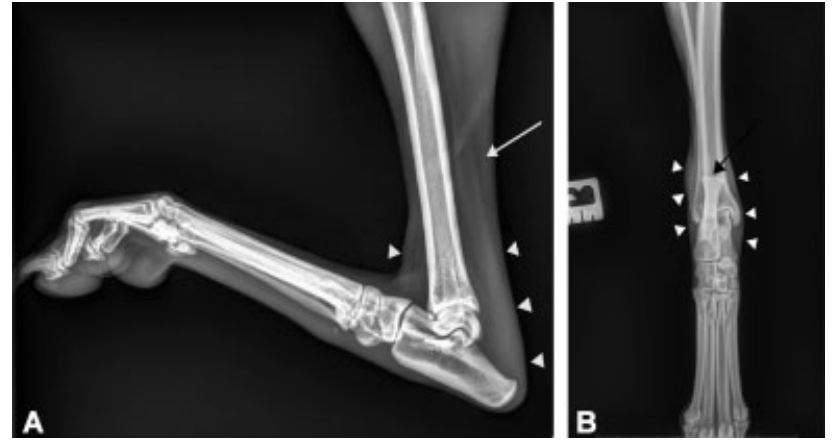

Fig. 1 Radiographs of the tarsus in two orthogonal projections. (A) Mediolateral projection: increased soft tissue radio-opacity surrounding the tarsus and the distal third of the Achilles tendon (AT) (white arrow heads) and AT thinning at the middle third of the tibia (white arrow). (B) Dorsoplantar projection: soft tissue swelling around the distal third of the tibia (white arrowheads) and a shallow superficial digital flexor tendon groove on the calcaneal tuberosity (black arrow).

examination did not disclose additional pathological findings. Observation from a distance revealed non-weightbearing lameness of the right pelvic limb. Thickness change and swelling over the calcaneal tendon were detected on palpation as well as swelling on both sides of the calcaneus. Tibiotarsal hyperflexion with the stifle extended was elicited and there was no pain, crepitus, or instability on tarsal manipulation. Neurological examination was unrevealing. Blood profile was also unremarkable.

Radiographs performed to visualize the tarsus and rule out fractures revealed soft tissue swelling around the distal AT end and a shallow SDFT groove on the calcaneal tuberosity, but no bone fractures (-Fig. 1). Ultrasonography of the right pelvic limb was performed to assess the integrity of the various AT components and showed two clear ruptures involving different fascicles of the calcaneal tendon situated at 6 and $1 \mathrm{~cm}$ from the calcaneal tuber, respectively. The SDFT luxation was also suspected by ultrasound. Based on this diagnosis, surgical treatment was indicated.
A caudolateral approach to the right AT was undertaken with a skin incision extending from the mid-tibia to the calcaneus. Two AT components ruptures were found: a partial tear of the gastrocnemius tendon situated $1 \mathrm{~cm}$ from the calcaneal tuber and a complete tear of the common tendon of the biceps femoris, gracilis, and semitendinosus muscles at $6 \mathrm{~cm}$ from the calcaneal tuber (-Fig. 2A). The SDFT was found intact, albeit laterally luxated and its medial retinaculum torn ( - Fig. 2B). Tenorrhaphy of the two tendon ruptures was performed by means of a locking loop tendon suturing technique using 2-0 polypropylene (Prolène, Ethicon Inc., Georgia, United States) sutures ( - Fig. 2B and C) after gentle debridement. A cortical calcaneo-tibial screw was subsequently inserted. Reduction in the laterally luxated SDFT followed. The tendon proved irreducible at first, yet a releasing incision in the lateral aspect of the calcaneal bursa and retinaculum enabled it eventually; some of the redundant bursa and retinaculum tissue were excised. The reduction was stabilized by several cruciate interrupted sutures placed medially in the retinaculum using a 2-0 polypropylene (Prolène, Ethicon Inc., Georgia, United States) suture ( - Fig. 2C). After suturing the retinaculum, a manual pressure was exerted on SDFT (laterally and medially distinctly) while rotating metatarsus medially and flexing tarsus to exclude reluxation.

Postoperative radiographs confirmed the appropriate position of the calcaneo-tibial screw. To immobilize and unload the repaired tendon, a casting bandage was applied.

Intravenous (IV) fluids (Ecoflac Ringer's lactate injectable solution, Virbac France, Carros Cedex, France), methadone (Comfortan, Dechra Veterinary Products SAS, Montigny-leBretonneux, France) $(0.2 \mathrm{mg} / \mathrm{kg}$ IV q. 4 hours $)$, and amoxicillin/clavulanate (Augmentin, Laboratoire GlaxoSmithKline, Rueil-Malmaison, France) ( $20 \mathrm{mg} / \mathrm{kg}$ IV q. 6 hours) were continued for 24 hours postoperatively, at which point the dog was discharged with the following oral medication: meloxicam (Metacam, Boehringer Ingelhheim, Reims CEDEX,
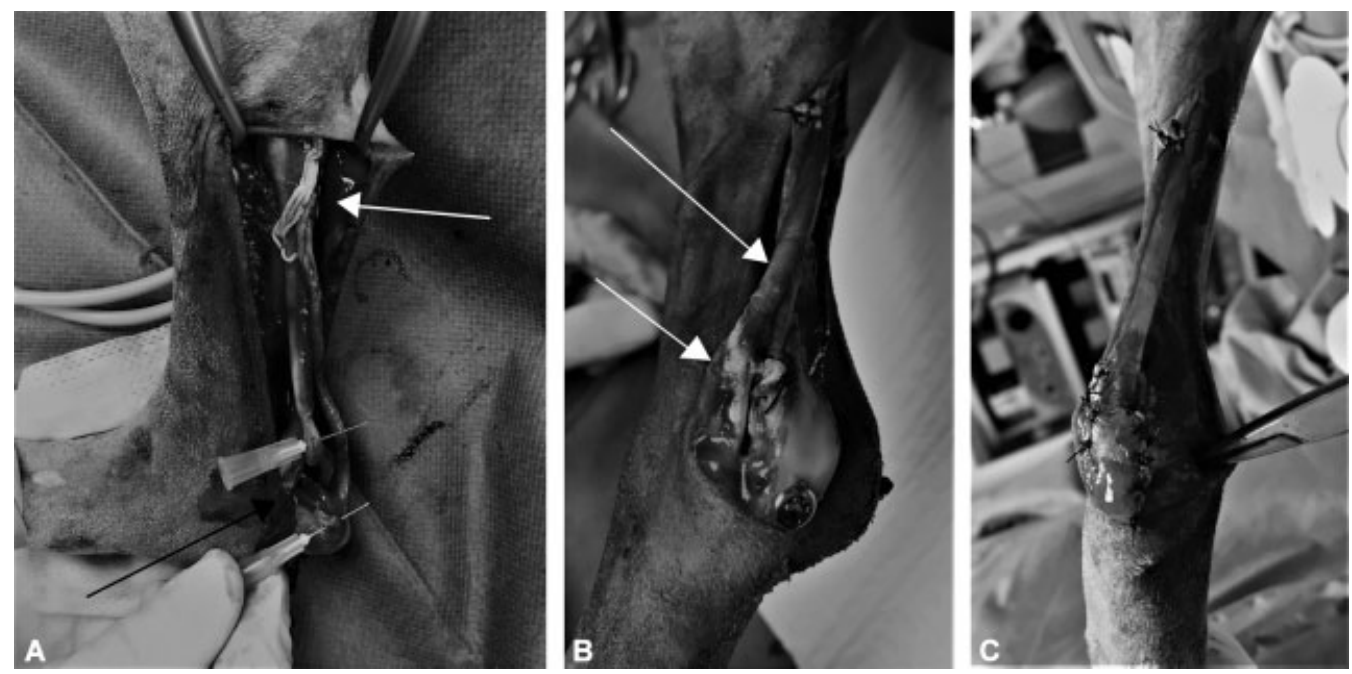

Fig. 2 Intraoperative photographs. (A) Partial rupture of the Achilles tendon (AT) situated 1 (black arrow) and $6 \mathrm{~cm}$ (white arrow) from the calcaneal tuber (B) View of the superficial digital flexor tendon lateral luxation (white arrows), head of the calcaneo-tibial screw and tendon sutures on the partial AT rupture. (C) View of the tendon sutures and the retinaculum sutures. 
France) $0.1 \mathrm{mg} / \mathrm{kg}$ once daily for 10 days and tramadol (Tralieve, Dechra Veterinary Products SAS, Montigny-le-Bretonneux, France) $4 \mathrm{mg} / \mathrm{kg}$ q. 8 hours for 5 days.

Three weeks after surgery, the dog had moderate lameness. Cast removal revealed a calcaneal pressure sore with screw exposition having been caused by the casting bandage, while the integrity of the tendon seemed preserved on palpation. Tarsal radiographs showed soft tissue swelling around the tarsus and continuity of the AT. Under these circumstances, screw removal was decided and once performed, the tarsus remained stable, hock hyperflexion unfeasible, and the SDFT non-luxating.

Wound management ensued as follows: regular wound dressing (q. 2 days) first with honey for 6 days and then with hydrogel (Intrasite, Smith \& Nephew SAS, Neuilly-sur-Seine, France), non-adhesive hydrocellular foam dressing (Allevyn, Smith \& Nephew SAS, Neuilly-sur-Seine, France), a doughnut dressing to reduce pressure applied onto the wound, and a protective bandage.

Significant lameness improvement ensued 8 weeks postoperatively and was assessed by two recheck appointments at 4 and 8 weeks post surgery, followed by mild intermittent lameness after exercise, and finally no lameness by the 20th week after surgery reported by phone call.

\section{Discussion}

The clinical signs of AT injury are mechanical lameness involving a plantigrade gait of the affected limb and swelling around the tendon. ${ }^{1}$ In partial AT rupture, the SDFT is often unaffected; therefore, hyperflexion of the digits occurs, resulting in a crab claw stance, along with tibiotarsal hyperflexion, and varying degrees of plantigrade stance. ${ }^{4}$ In this reported case, digit hyperflexion was absent despite partial AT rupture and intact SDFT because the latter was simultaneously luxated. Moreover, the characteristic feeling of SDFT 'luxation-reduction' was absent, probably due to the permanent nature of the luxation and/or the concomitant partial AT rupture. Furthermore, the initial orthopaedic examination revealed swelling on both sides of the calcaneus, which suggested SDFT luxation as described by Gatineau and Dupuis. $^{5}$

Ultrasonography is mandatory as part of the examination and diagnosis in cases of tendon and muscle injuries. ${ }^{3,6}$

Tendon healing is a lengthy process and complications like diastasis may occur. ${ }^{1}$ Nonetheless, tendon healing requires some degree of strain along the length of the tendon to stimulate proper recovery because strength and alignment of collagenous and scar tissue formation are positively affected by exercise and lessened with immobilization. ${ }^{7}$

Various reconstruction techniques have been employed in instances of tendon rupture with loss of tendon length and inability to approximate tendon end, ${ }^{1,3}$ that is, (1) autologous grafts, (2) heterologous implants, and (3) synthetic materials. These techniques are more typically necessary in chronic AT ruptures.

During postoperative management, primarily in the early stages of healing, limb immobilization is essential, whether in cases of AT rupture or SDFT luxation. Immobilization is implemented after AT rupture repair to diminish tensile forces exerted on sutures, notwithstanding that tibiotarsal immobilization in the dog does not eliminate traction and has no effect on maximal traction strength of the AT in weight-bearing. ${ }^{7}$ The benefit of immobilization seems to result rather from weight-bearing reduction in the immobilized limb and exercise restriction. ${ }^{7}$ Several joint immobilization techniques have been employed in AT rupture, for example, transarticular external skeletal fixator, calcaneotibial bone screw placement with or without a casting wrap, and various configurations of casts and splints. ${ }^{7-9} \mathrm{~A}$ study by Nielsen and Pluhar compared immobilization methods but found no significant differences between them in terms of complication rate, duration of immobilization, recovery time or functional outcome. ${ }^{8}$ Immobilization by calcaneo-tibial bone screw and a casting bandage was done in our case since this combined method has proved to yield better results than casting alone. ${ }^{10}$ Nonetheless, every immobilization technique is liable to potential complications ${ }^{7-9}$ and, in light of the present dog's breed, the casting bandage placed for additional safety on top of the calcaneo-tibial screw may have been obviated; indeed, sighthounds have higher pressure point sensitivity to casting. A cranial cast could have been an alternative. ${ }^{9}$ In the combined screw-and-cast immobilization method, the casting bandage may be maintained for 2 to 4 weeks, whereas the calcaneo-tibial screw is usually withdrawn 6 to 8 weeks postoperatively. ${ }^{3,4}$ A premature screw removal had to be resorted to in our case because of both the screw exposure and the risk of infection through the cast-inflicted pressure sore.

The prescribed postoperative follow-up was adhered to by the owner and a full functional recovery ensued. The outcome of surgically treated AT rupture in dogs is good ${ }^{3}$ despite the long rehabilitation period of approximately 20 weeks. ${ }^{1}$ The prognosis is also excellent in SDFT luxation provided that tibiotarsal immobilization is adequately performed and surgical repair performed before fibrosis sets in. ${ }^{2}$ This case report describes successful management of a partial rupture of AT (partial tear of the gastrocnemius tendon and complete tear of the tendon group formed by the biceps femoris, gracilis, and semitendinosus muscles) and concomitant SDFT luxation.

\section{Conclusion}

The combined partial AT rupture and SDFT lateral luxation has not been hitherto reported. As the prognosis of these two pathologies depends on tendon healing and immobilization, and since their postoperative care is similar, they were treated conjointly and the long-term functional postoperative outcome proved highly satisfactory.

Authors Contributions

Claire Deroy-Bordenave performed the surgery and revised the manuscript. Hélène Dosseray assisted on the surgery, wrote, and submitted the manuscript. 
e40 Concomitant Partial AT Rupture and Lateral Luxation of the SDFT in a Dog Dosseray and Deroy-Bordenave

\author{
Funding \\ None.

\section{Conflicts of Interest} \\ None declared.
}

\section{References}

1 Spinella G, Tamburro R, Loprete G, Vilar JM, Valentini S. Surgical repair of Achilles tendon rupture in dogs: a review of the literature, a case report and new perspectives. Vet Med (Praha) 2010; 55:303-310

2 Solanti S, Laitinen O, Atroshi F. Hereditary and clinical characteristics of lateral luxation of the superficial digital flexor tendon in Shetland sheepdogs. Vet Ther 2002;3(01):97-103

3 Corr SA, Draffan D, Kulendra E, Carmichael S, Brodbelt D. Retrospective study of Achilles mechanism disruption in 45 dogs. Vet Rec 2010;167(11):407-411

4 Harasen G. Ruptures of the common calcaneal tendon. Can Vet J 2006;47(12):1219-1220
5 Gatineau M, Dupuis J. Longitudinal tendon tear concurrent with bilateral medial luxation of the superficial digital flexor muscle tendon in a dog. Vet Comp Orthop Traumatol 2010;23(04): 289-293

6 Kramer M, Gerwing M, Michele U, Schimke E, Kindler S. Ultrasonographic examination of injuries to the Achilles tendon in dogs and cats. J Small Anim Pract 2001;42(11):531-535

7 Lister SA, Renberg WC, Roush JK. Efficacy of immobilization of the tarsal joint to alleviate strain on the common calcaneal tendon in dogs. Am J Vet Res 2009;70(01):134-140

8 Nielsen C, Pluhar GE. Outcome following surgical repair of Achilles tendon rupture and comparison between postoperative tibiotarsal immobilization methods in dogs: 28 cases (1997-2004). Vet Comp Orthop Traumatol 2006;19(04):246-249

9 Sugiyama T, Woodward A, Ryan SD. Biomechanical evaluation of 6 transarticular tibiotarsal immobilization methods in canine cadaveric limbs. Vet Surg 2018;47(05):705-714

10 Worth AJ, Danielsson F, Bray JP, Burbidge HM, Bruce WJ. Ability to work and owner satisfaction following surgical repair of common calcanean tendon injuries in working dogs in New Zealand. N Z Vet J 2004;52(03):109-116 